\title{
An Improved Five Class MI Based BCI Scheme for Drone Control Using Filter Bank CSP
}

Christensen, Søren Møller; Holm, Nicklas Stubkjær; Puthusserypady, Sadasivan

Published in:

Proceedings of 2019 7th International Winter Conference on Brain-Computer Interface

Link to article, DOI:

10.1109/IWW-BCI.2019.8737263

Publication date:

2019

Document Version

Peer reviewed version

Link back to DTU Orbit

Citation (APA):

Christensen, S. M., Holm, N. S., \& Puthusserypady, S. (2019). An Improved Five Class MI Based BCI Scheme for Drone Control Using Filter Bank CSP. In Proceedings of 2019 7th International Winter Conference on BrainComputer Interface IEEE. https://doi.org/10.1109/IWW-BCI.2019.8737263

\section{General rights}

Copyright and moral rights for the publications made accessible in the public portal are retained by the authors and/or other copyright owners and it is a condition of accessing publications that users recognise and abide by the legal requirements associated with these rights.

- Users may download and print one copy of any publication from the public portal for the purpose of private study or research.

- You may not further distribute the material or use it for any profit-making activity or commercial gain

- You may freely distribute the URL identifying the publication in the public portal 


\section{An Improved Five Class MI Based BCI Scheme for Drone Control Using Filter Bank CSP}

\author{
Søren Møller Christensen \\ Department of Electrical Engineering \\ Technical University of Denmark \\ Lyngby, Denmark \\ s153571@ student.dtu.dk
}

\author{
Nicklas Stubkjær Holm \\ Department of Electrical Engineering \\ Technical University of Denmark \\ Lyngby, Denmark \\ s154411@ student.dtu.dk
}

\author{
Sadasivan Puthusserypady \\ Department of Electrical Engineering \\ Technical University of Denmark \\ Lyngby, Denmark \\ spu@elektro.dtu.dk
}

\begin{abstract}
Worldwide, millions of people are locked in or in a wheelchair, due to several neuromuscular disorders or spinal cord injuries. These individuals are deprived of trivial social activities, like interacting or playing games with other people. Such activities are crucial for personal development, and can have a great impact on the quality of their lives. This work aims at the design and implementation of an electroencephalography (EEG) based motor imagery (MI) brain computer interface (BCI) system that would allow disabled, and able-bodied, individuals alike to control a drone in a 3D physical environment by only using their thoughts. An improved version of the filter bank common spatial pattern (FBCSP) algorithm was developed, and it has shown to perform superior $(68.5 \%$ accuracy) to the winning FBCSP algorithm (67.8\% accuracy), when tested on dataset 2a (4 class MI) of the BCI competition IV. A deep convolutional neural network (CNN) based algorithm was also implemented and tested on the same dataset, which however performed inferior (62.9\% accuracy) to the winner, as well as our proposed FBCSP algorithms. The improved FBCSP was then tested on our inhouse 5-class (left hand, right hand, tongue, both feet and rest) MI dataset (collected from 10 able-bodied subjects) and obtained a mean accuracy of $41.8 \pm 11.74 \%$. This is considered a significant result though it is not good enough to attempt the control of a real drone.
\end{abstract}

Index Terms-Brain Computer Interface (BCI), Motor Imagery (MI), Filter Bank Common Spatial Pattern (FBCSP), Convolutional Neural Network (CNN), Drone Control, Multiclass Motor Imagery.

\section{INTRODUCTION}

According to the World Health Organization (WHO), 2-4\% of the World's population is suffering from severe disability and/or paralysis [1]. The global estimation is on the rise due to an aging population, and rapid spread of chronic diseases. Common causes of paralysis includes stroke, multiple sclerosis, spinal cord injury, cerebral palsy, and many more [2]. Some of these individuals are "locked-in", rendering them unable to speak or move.

Brain Computer Interface (BCI) systems establish a direct connection between the brain and a computer, thereby creating a communication channel that bypasses the peripheral nervous system [3]. BCI utilizes neurofeedback by acquiring and processing electroencephalogram (EEG) signals to control external devices based on the individual's wishes. Steady State Visual Evoked Potentials (SSVEP), Event Re- lated Potentials (ERPs), and Event Related Desynchronization/Synchronization (ERD/ERS) components as well as sensorimotor rhythms (SMRs) are the most commonly exploited EEG responses in BCI systems [4] [5] [6]. SMRs are tuned by motor intentions, such as motor imagery (MI), and are characterized by a modulation of the amplitudes of the measured electrical potentials [7].

Even though both SSVEP and P300 based BCIs have shown to reach high accuracies, they both use flashing visual stimuli, which subjects have reported to cause fatigue after long usage [8] [9]. Both SSVEP and P300 also requires that the subject is able to at least move their eyes, which may not be the case for all patients [10]. The MI based BCI system on the other hand requires only the imagined movement of the limbs, and therefore is the most appropriate chosen paradigm for lockedin patients [7].

In recent years, there have been many attempts to improve the MI based BCI systems. One of the main reasons for this has been the availability of the BCI competition database, which made it easier for researchers to experiment with their own algorithms [11]. One of the most popular datasets is dataset $2 \mathrm{a}$ (4-class $\mathrm{MI}$ ) from BCI competition IV. The winner of this competition on dataset $2 \mathrm{a}$ scored an accuracy of $67.8 \%$ using the Filter Bank Common Spatial Pattern (FBCSP) algorithm [12]. This result was beaten by a group achieving $70 \%$ mean accuracy utilizing multivariate empirical mode decomposition based filtering and Riemannian geometry for classification [13]. In 2017, Sharbaf, et al., improved this accuracy (72.3\%) considerably by implementing the Filter Bank Common Spatio-Spectral Patterns (FBCSSP), with variable sized frequency bands and a channel selection algorithm [14]. In the same year, the work by Schirrmeister et al., have reportedly beaten that record by $1.5 \%(73.7 \%)$ using a shallow end-to-end Convolutional Neural Network (CNN) based algorithm [15]. Later in the same year, a project sponsored by the US military and UK ministry of defence, used an end-to-end deep CNN that have claimed to have achieved an accuracy of $81.1 \%$ [16]. Recently, a group claimed to have achieved an accuracy of $88.5 \%$ using a 1D shallow CNN [17].

Lafleur et al., have developed a 4-class MI BCI setup to 
control a drone using a 64 channel EEG system and have achieved an accuracy of $90.5 \%$ [18]. This system was however tested on only one subject. Here, we propose the development and implementation of a 5-class MI based BCI system, that is capable of controlling a drone in 3D space, by utilizing forward, clockwise and anticlockwise rotation, and up and down movement, the proposed drone control scheme could be of use to paralyzed and able-bodied individuals alike in the future.

\section{A. Our Work}

We have implemented a modified version of the FBCSP algorithm where we have split a certain frequency range into a number of bands using grid search, unlike the manual selection, nor did we utilize a feature selection algorithm as in its original version [12] [19] [20]. We use a support vector machine (SVM) classifier to achieve superior results compared to the winner FBCSP algorithms results [12] [21]. A deep convolutional neural network (CNN) based algorithm with five hidden layers was also implemented in this project, which however performed inferior to the winner [12], as well as our improved FBCSP algorithm.

\section{MATERIALS AND MethodS}

\section{A. Common Spatial Pattern Filter}

The purpose of a CSP filter, is to identify the sources of neuro-electric signals for distinguishing between different classes or populations (e.g., MI of left and right hands). The CSP algorithm is described mathematically, by first estimating the covariance matrices [22]. Let $\mathbf{X} \in \mathbb{R}^{N_{c h} \times N}$ be the recorded EEG signal, where $N$ is the number of samples and $N_{c h}$ is the number of EEG channels. Then, the filtered signal, $\mathbf{Z}_{C S P} \in \mathbb{R}^{N_{c h} \times N}$ can be represented as:

$$
\mathbf{Z}_{C S P}=\mathbf{W}^{T} \mathbf{X}
$$

where $\mathbf{W} \in \mathbb{R}^{N_{c h} \times N_{c h}}$ is the matrix parameterizing the signal decomposition, which projects the recorded EEG signal from the sensor to the source spatial locations. Here, we denote each column $\left(\mathbf{w}_{i}, i=1,2, \ldots, N_{c h}\right)$ of $\mathbf{W}$ as a spatial filter, and each column of $\mathbf{W}^{-1}$ as a spatial pattern.

Assuming a two-class ( $a$ and $b$ ) problem, the basic principle behind the CSP algorithm is to maximize the variance of one class while minimizing it for the other. This is achieved by operating on the respective covariance matrices $\left(\mathbf{R}_{a}, \mathbf{R}_{b}, \in\right.$ $\left.\mathbb{R}^{N_{c h} \times N_{c h}}\right)$.

$$
\begin{aligned}
\mathbf{R}_{a} & =\frac{1}{N_{a}} \sum_{n=1}^{N_{a}} \frac{\mathbf{X}_{a}^{n} \mathbf{X}_{a}^{n T}}{\operatorname{Tr}\left(\mathbf{X}_{a}^{n} \mathbf{X}_{a}^{n T}\right)} \\
\mathbf{R}_{b} & =\frac{1}{N_{b}} \sum_{n=1}^{N_{b}} \frac{\mathbf{X}_{b}^{n} \mathbf{X}_{b}^{n T}}{\operatorname{Tr}\left(\mathbf{X}_{b}^{n} \mathbf{X}_{b}^{n T}\right)}
\end{aligned}
$$

Here, $N_{a}$ and $N_{b}$ are the number of trials of class $a$ and class $b$, respectively. $\mathbf{X}_{a}^{n}$ and $\mathbf{X}_{b}^{n}$, both $\in \mathbb{R}^{N_{c h} \times N}$ are the EEG data for the $n^{\text {th }}$ trial of class $a$ and class $b$ and $\operatorname{Tr}($. denotes the trace operation of the matrix.
These two covariance matrices are combined to obtain the covariance matrix $\mathbf{R}_{c}=\mathbf{R}_{a}+\mathbf{R}_{b}$ and subsequently the eigen decomposition of it (i.e., $\mathbf{R}_{c}=\mathbf{U}_{c} \boldsymbol{\Lambda}_{c} \mathbf{U}_{c}^{T}$ ) is performed to construct a whitening matrix $\mathbf{P}$ as follows:

$$
\mathbf{P}=\sqrt{\boldsymbol{\Lambda}_{c}^{-1}} \mathbf{U}_{c}^{T}
$$

where, $\mathbf{U}_{c}$ is the matrix composed of eigenvectors and $\boldsymbol{\Lambda}_{c}$ is the matrix of eigenvalues of $\mathbf{R}_{c}$. Using this matrix $\mathbf{P}$, the covariance matrices $\mathbf{R}_{a}$ and $\mathbf{R}_{b}$ are whitened (i.e., $\mathbf{S}_{a}=\mathbf{P} \mathbf{R}_{a} \mathbf{P}^{T}$ and $\mathbf{S}_{b}=\mathbf{P} \mathbf{R}_{b} \mathbf{P}^{T}$ ) such that $\mathbf{S}_{a}+\mathbf{S}_{b}=$ $\mathbf{P} \mathbf{R}_{c} \mathbf{P}^{T}=\mathbf{I}$, where $\mathbf{I}$ is the identity matrix. Further, the eigen decomposition of $\mathbf{S}_{a}$ and $\mathbf{S}_{b}$ provide the following:

$$
\begin{aligned}
\mathbf{S}_{a} & =\mathbf{B} \Phi_{a} \mathbf{B}^{T}, \\
\mathbf{S}_{b} & =\mathbf{B} \Phi_{b} \mathbf{B}^{T} .
\end{aligned}
$$

Here, $\boldsymbol{\Phi}_{a}$ and $\boldsymbol{\Phi}_{b}$ are the matrix of eigenvalues (arranged in descending order) corresponding to classes $a$ and $b$, respectively. The matrix $\mathbf{B}$ can now be used to optimize the variation between classes $a$ and $b$, by finding the spatial filters, $\mathbf{V} \in \mathbb{R}^{N_{c h} \times N_{c h}}$, where each column is a filter.

$$
\mathbf{V}=\mathbf{B}^{T} \mathbf{P} \text {. }
$$

From V, $2 m$ filters ( $m$ filters from column 1 to column $m$ and $m$ filters from column $N_{c h}-m$ to column $N_{c h}$ ) are selected to obtain the spatial filter $\tilde{\mathbf{W}} \in \mathbb{R}^{N_{c h} \times 2 m}$. The signal matrix $\mathbf{X}$ is then transformed as follows:

$$
\tilde{\mathbf{Z}}_{C S P}=\tilde{\mathbf{W}}^{T} \mathbf{X} \text {. }
$$

The feature vector, $\mathbf{f}_{n}$ for the $n^{\text {th }}$ trial for classification can now be calculated as:

$$
\mathbf{f}_{n}=\log \left(\frac{\operatorname{diag}\left(\tilde{\mathbf{W}}^{T} \mathbf{X}^{n} \mathbf{X}^{n T} \tilde{\mathbf{W}}\right)}{\operatorname{Tr}\left(\tilde{\mathbf{W}}^{T} \mathbf{X}^{n} \mathbf{X}^{n T} \tilde{\mathbf{W}}\right)}\right)
$$

where $\operatorname{diag}(\cdot)$ denotes the diagonal elements of the resulting matrix.

CSP is an algorithm that only works for binary classes, since it can only maximize the variance between two classes. However, there have been attempts to extend this to multiple classes, such as, the one-versus-one (OVO) and the one-versusrest (OVR) algorithms, that have been implemented in this work [12] [22] [23].

\section{B. Filter Bank CSP}

In FBCSP, the CSP has been extended to have multiple frequency bands. The main idea is to filter the data using a number of subband filters in the frequency band, that contains the MI information. There are different ways of doing this: In [12], they manually selected 9 bands from 4-40Hz. Following this approach in our work, we however choose the frequency range to be $4-34 \mathrm{~Hz}$ for our winning settings. This frequency band is divided into $N_{s b}$ sub-bands. After filtering the signal with these filters, the $N_{s b}$ CSP filter sets and corresponding features are computed. When a CSP multi-class extension is used simultaneously with the filter bank, we get $N_{s b} \times N_{c l} \times$ $N_{c s p}$ features, where $N_{c l}$ is the number of classifiers, and 
$N_{c s p}$ is the number of CSP features to calculate (equal to $2 m$ in CSP).

\section{Convolutional Neural Networks}

CNN's have in recent years been very successful in various fields like image recognition, data mining, and signal processing [24] [25] [26]. In this work, we implemented almost the same architecture as the one suggested by Zhao et al. [16]. However this is a general network, trained on all subjects. We used one fully connected layer followed by $5 \mathrm{CNN}$ layers. Batch normalization was done to speed up the training of the network, and 50\% dropout was implemented after each CNN layer for preventing overfitting, and finally activation layers was implemented for the nonlinear relationship [27] [28]. The rectified linear unit (ReLU) was used as activation layer instead of exponential linear unit (ELU) as this provided us better results [29] [30]. For down sampling, we have used max pooling.

\section{Experimental Setup and Data Details}

We used two datasets for evaluating the proposed algorithms: (i) the open source dataset (dataset 2a, 4-class MI of different body parts from BCI competition IV), and (ii) an inhouse dataset (5-class MI) of different body parts, which were recorded and evaluated in real-time.

\section{A. Dataset 1: 4-class MI}

This dataset belongs to the BCI competition IV (dataset 2a) and comprises of the EEG recordings on 9 subjects of 4classes of MI from distinct body parts (left, right hand, feet and tongue) [11]. For each subject, two sessions of 288 trials were recorded, namely a calibration session without feedback and an evaluation session with feedback. 25 electrodes (3 EOG channels and 22 EEG channels) were used for the recordings of this dataset.

\section{B. Dataset 2: 5-class MI}

The second dataset was collected from our own laboratory. The 5 classes of MI data include left and right hand, both feet, rest and tongue. These 5 classes corresponds too counterclockwise, clockwise, upward, downward and forward movement of a drone.

The 5 classes were selected as the proposed classes for controlling a drone in the future.

For the experiment, a total of 160 training trials were recorded (32 trials for each class), of which the first 60 training trials were recorded without feedback, and the remaining 100 training trials were recorded with feedback in real-time. For evaluation, 100 trials were recorded, where feedback was also given to the subjects in real-time. During training and evaluation, the trials were split into sessions (each of 20 trials), where the subject were given the opportunity to rest for a few minutes in between sessions.

The experiments were carried out in a soundproof and electromagnetically shielded room. A total of 10 able-bodied subjects ( 3 females and 7 males in the age range of 2025 years) participated in the experiment. All subjects were provided verbal informed consent prior to their participation in the experiments, which were approved by the Regional Committee on Health Research Ethics for the Capital Region of Denmark (reference H-3-2013-004) and carried out in accordance with the corresponding guidelines and relevant regulations on the use of human subjects for health-related scientific research.

The subjects were seated in a comfortable chair, and the computer screen was placed about $60 \mathrm{~cm}$ in front of them. For recording the signals, the g.USBamp amplifier was used with 16 electrodes (16 EEG channels in the motor cortex area), a reference electrode and a ground electrode (Fig.1 [31]). The sampling rate was set to $512 \mathrm{~Hz}$, and for preprocessing of the signals, an 8th order Butterworth band pass $(2-60 \mathrm{~Hz})$ filter and a 4th order Butterworth notch filter to suppress the power-line interference were chosen. An Acer Aspire 5 computer (8th generation Intel processor (i5-8250U), Nvidia graphics card (MX150) and a full HD screen) were used for the experiment.

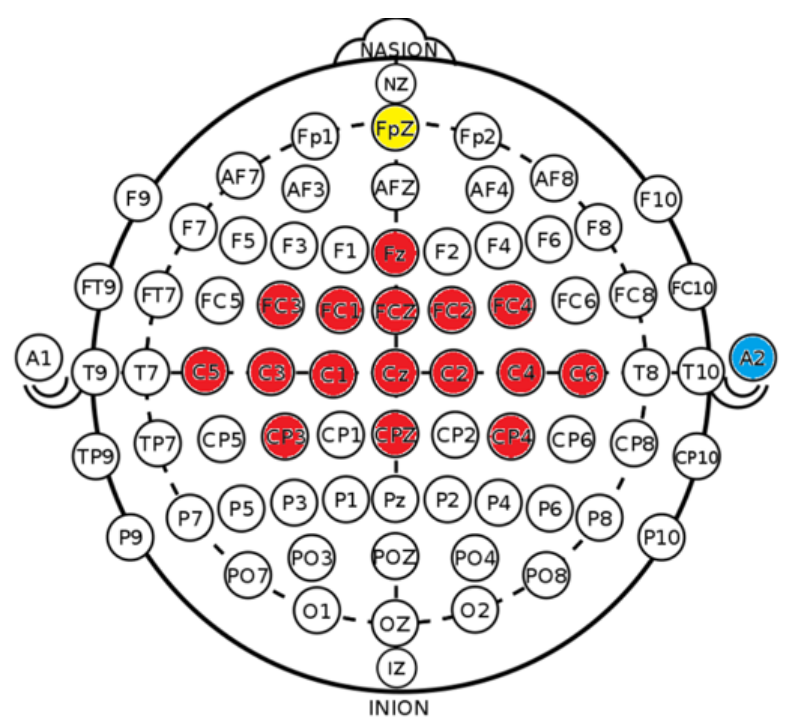

Fig. 1. Placement of electrodes during recordings. Red indicate the chosen electrodes, yellow is the ground and blue is the reference.

\section{Timing Scheme}

The timing scheme for each trial is shown in Fig.2. Each trial began with the participant gazing at a fixation cross at the center of the screen for 2 seconds. This was followed by a break of 0.5 seconds, before the MI period of 4 seconds began, where the cue was present throughout the MI period. If the subject was training with feedback or an evaluation trial was recorded, then feedback was given to the subject after 2 seconds, otherwise no feedback was given. Afterwards there was a varying break time of 1-2 seconds. Using this timing scheme, the average trial took about 8 seconds.

\section{Feedback}

Feedback is a very important part of the visual interface as it lets the subject know how he/she is performing in real-time. 


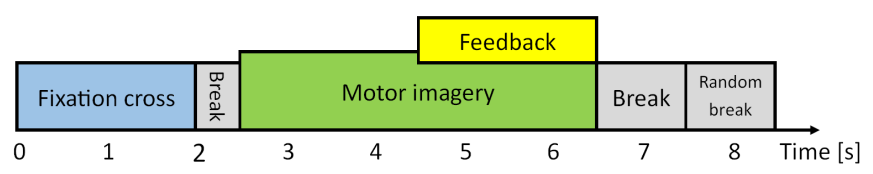

Fig. 2. Timing scheme for data recording. For training trials recorded without feedback, the timing scheme is the same, but the feedback is omitted.

By implementing feedback during training of the classifier, both the classifier and the subject is trained, so they together can achieve a higher performance.

During our recordings, the feedback was given to the subject in two ways: (i) a green box was shown during the MI period, if the classifier was correctly classifying the trial. The green box was only shown 2 seconds into the MI period and until the end of the MI period, as can be seen in Fig.2. This was done since the first few predictions are only based on few windowed trials, and therefore the result of classification had a tendency to vary more in the beginning. (ii) Feedback in the form of an accuracy score was given at the end of each session, so the subject could see if he/she had any progress during the last session.

\section{RESUlts AND Discussion}

The performance (classification accuracy) of the proposed methods are provided in this section.

\section{A. Dataset 1: 4-Class MI}

Table I shows the accuracies obtained for the 9 subjects on the 4-class dataset $2 \mathrm{a}$ from BCI competition IV for the proposed modified FBCSP and CNN algorithms. It also shows the corresponding accuracies for the competition winner [22] as well as the 1st and 2nd runner-up [32]. It can be seen that our modified FBCSP algorithm perform superior $(68.5 \%)$ to the 2008 winner $(67.8 \%)$. Its performance is much better than the first and second runner up. It may be noted that out of the 9 subjects, the performances for four subjects (subjects 1, 2, 4 and 5) are significantly higher in our proposed FBCSP than the performances of these subjects with the BCI competition IV winner. The average accuracy using the CNN algorithm is seen to be inferior to our algorithm, the 2008 winner and the 1 st runner-up. The performance of subject $3(83.0 \%)$ however, is the highest among all.

\section{B. Dataset 2: 5-Class MI}

The results (classification accuracies) for the modified FBCSP algorithm for the 5-class MI data collected from the 10 subjects in our experiment are presented in Table II. The mean accuracy is $41.80 \pm 11.74 \%$, which does not seem as much, but is much higher than the chance level of $20 \%$, and the accuracies given in Table II have been calculated in real-time, to reflect the true performance of the algorithm. It may be noted that the score of subject 5 is significantly lower $(15 \%)$ than the average. If this subject is omitted from the analysis, the average would increase (And the standard deviation would
TABLE I

CLASSIFICATION ACCURACIES (\%) FOR THE PROPOSED MODIFIED FBCSP AND CNN ALGORITHMS ALONG WITH THE BCI COMPETITION IV WINNER AND THE 1ST AND 2ND RUNNERS-UP FOR DATASET 2A. RESULTS FOR THE WINNER, AND THE 1ST AND 2ND RUNNERS UP WERE FOUND ON THE OFFICIAL WEBSITE OF BCI COMPETITION IV [32].

\begin{tabular}{c|l|l|l|l|l}
\hline Sub. & $\begin{array}{l}\text { This } \\
\text { work } \\
\text { (FBCSP) }\end{array}$ & $\begin{array}{l}2012 \\
\text { Winner } \\
{[22]}\end{array}$ & $\begin{array}{l}\text { 1st } \\
\text { Runner } \\
\text { up [32] }\end{array}$ & $\begin{array}{l}\text { This } \\
\text { work } \\
\text { (CNN) }\end{array}$ & $\begin{array}{l}\text { 2nd } \\
\text { Runner } \\
\text { up [32] }\end{array}$ \\
\hline \hline 1 & $\mathbf{8 1 . 9}$ & 76.0 & 76.8 & 71.2 & 53.5 \\
2 & $\mathbf{5 8 . 0}$ & 56.5 & 50.5 & 35.4 & 38.5 \\
3 & 75.7 & 81.3 & 78.3 & $\mathbf{8 3 . 0}$ & 61.0 \\
4 & $\mathbf{6 4 . 2}$ & 61.0 & 58.0 & 52.1 & 49.8 \\
5 & $\mathbf{6 2 . 8}$ & 55.0 & 37.0 & 49.0 & 30.3 \\
6 & 45.1 & $\mathbf{4 5 . 3}$ & 40.8 & 51.4 & 35.5 \\
7 & 81.9 & $\mathbf{8 2 . 8}$ & 74.5 & 78.8 & 46.8 \\
8 & 77.1 & $\mathbf{8 1 . 3}$ & 79.8 & 77.8 & 61.8 \\
9 & 69.4 & 70.8 & $\mathbf{7 6 . 8}$ & 67.7 & 58.0 \\
\hline \hline Avg. & $\mathbf{6 8 . 5}$ & 67.8 & 63.6 & 62.9 & 49.0 \\
\hline Std. & 12.2 & 13.7 & 17.2 & 16.5 & 11.5 \\
\hline & \multicolumn{5}{|l}{}
\end{tabular}

decrease considerably) to $44.78 \pm 7.43 \%$. Each subject had 100 evaluation trials. Here, the $\mathrm{CNN}$ algorithm was not used as its performance on Dataset 1 proved to be inferior to both the winner and our algorithm.

TABLE II

CLASSIFICATION ACCURACIES (\%) OF MODIFIED FBCSP ALGORITHM FOR THE 10 SUBJECTS FOR THE 5-CLASS MI DATA SET RECORDED IN OUR LABORATORY. ACCURACIES WERE CALCULATED USING 100 EVALUATION TRIALS, AND WERE CALCULATED IN REAL-TIME USING AN ONLINE VERSION OR OUR FBCSP ALGORITHM.

\begin{tabular}{c|c|c|c|c|c|c|c|c|c|c|c}
\hline Sub & 1 & 2 & 3 & 4 & 5 & 6 & 7 & 8 & 9 & 10 & Avg. \\
\hline \hline Acc & 45 & 42 & $\mathbf{5 7}$ & 47 & 15 & 35 & 35 & 43 & 45 & 54 & 41.8 \\
\hline Std & - & - & - & - & - & - & - & - & - & - & 11.74 \\
\hline
\end{tabular}

\section{Use OVO or OVR?}

From our analysis, it was found that OVO performed better than OVR when tested on Dataset 1 . The number of classifiers and filters to train differ in both these cases although both depends on the number of classes $\left(N_{\text {classes }}\right)$. The number of classifiers $\left(N_{c l}\right)$ required for OVR is equal to $N_{\text {classes }}$ and the number of classifiers for OVO is $N_{c l}=\frac{N_{\text {classes }}\left(N_{\text {classes }}-1\right)}{2}$. For 4-classes, these numbers are 4 and 6 , respectively for OVR and OVO methods. For 5-classes, these numbers are respectively, 5 and 10 (a doubling for OVO compared to OVR). A disadvantage of OVO (For a 5-class system), could be that for a given trial to be evaluated, only 4 of the classifiers have trained on trials belonging to that class, meaning that 6 of the classifiers voting, have not been trained on the relevant class.

\section{Future Improvements}

1) Further Training of Subjects: A study that includes 324 subjects performing 2-class MI concludes that MI is a skill that can be improved by practicing [33]. We have also experienced this while recording the signals from the subjects, who had no prior BCI experience. Imagining five different MI tasks is not easy, and therefore we believe that more training would have helped improve the accuracy significantly. 
2) Cross Fold Validation: Implementing cross fold validation on the training data, would be very beneficial. Cross fold validation could be used for testing different parameters manually, or for the program to automatically test different parameters using an optimization problem or a grid search. This could lead to an even more personalized classifier, as the parameters could then be tuned for the individual subject.

3) Adaptive Algorithm: An adaptive training algorithm might have resulted in better classifiers, by selecting which trials to train on, so trials which would lead to a lower accuracy, would not be trained on. Furthermore, an adaptive training algorithm, could calculate which classes the user is having problems imagining, and then increase the number of trials the user has to train on of those classes. This way, the user would get more experience in troubling classes, and thereby hopefully increasing the overall accuracy of the classifier [34].

\section{CONClusion}

This paper presents an improved version of the FBCSP method for classification of a 5-class MI BCI dataset. This method was first tested on the BCI competition IV dataset $2 \mathrm{a}$ and found that it beats the winner of the BCI competition by $0.7 \%$ ( $68.5 \%$ vs $67.8 \%)$. The proposed method was then applied on a 5-class MI dataset from 10 able-bodied subjects, which was collected in our laboratory using only 16 electrodes. The mean accuracy was found to be $41.8 \%$. This is considered a significant result considering the fact that the chance level is $20 \%$. However, this accuracy is not considered high enough to attempt to control a real drone.

\section{ACKNOWLEDGMENT}

The authors would like to thank the Hearing Systems laboratory at the Department of Electrical Engineering, DTU, for providing us the EEG facility for data collection. Special thanks to Bastian Epp, for continued support to use their EEG facility. We sincerely acknowledge the subjects who volunteered to participate in this study.

\section{REFERENCES}

[1] M. W. Chan and R. W. Zoellick, "World report on disability." [Online]. Available: http://www.who.int/disabilities/world_report/2011/report/en/

[2] NHS, "Paralysis." [Online]. Available: https://www.nhs.uk/conditions/paralysis/

[3] M. O. Krucoff, S. Rahimpour, M. W. Slutzky, V. R. Edgerton, and D. A. Turner, "Enhancing nervous system recovery through neurobiologics, neural interface training, and neurorehabilitation," Frontiers in Neuroscience, vol. 10, no. DEC, 2016.

[4] A. Kubacki and A. Jakubowski, "Classifier testing for the brain-machine interface (BCI) based on Steady State Visually Evoked Potential (SSVEP)," ITM Web of Conferences, vol. 15, 2017. [Online]. Available: https://www.itm-conferences.org/10.1051/itmconf/20171502003

[5] N. K. Squires, K. C. Squires, and S. A. Hillyard, "Two varieties of long-latency positive waves evoked by unpredictable auditory stimuli in man," Electroencephalography and Clinical Neurophysiology, vol. 38, no. 4, pp. 387-401, 1975.

[6] S. A. Huettel and G. McCarthy, "What is odd in the oddball task? Prefrontal cortex is activated by dynamic changes in response strategy," Neuropsychologia, vol. 42, no. 3, pp. 379-386, 2004.

[7] T. Mulder, "Motor imagery and action observation: Cognitive tools for rehabilitation," Journal of Neural Transmission, vol. 114, no. 10, pp. $1265-1278,2007$.
[8] C. G. C. Guan, M. Thulasidas, and J. W. J. Wu, "High performance P300 speller for brain-computer interface," in IEEE International Workshop on Biomedical Circuits and Systems, 2004., 2004, pp. 13-16.

[9] D. Makri, C. Farmaki, and V. Sakkalis, "Visual fatigue effects on Steady State Visual Evoked Potential-based Brain Computer Interfaces," in 7th International IEEE/EMBS Conference on Neural Engineering, NER 2015, vol. 2015-, 2015, pp. 70-73. [Online]. Available: http://www.scopus.com/inward/record.url?eid=2s2.0-84940371341\&partnerID=tZOtx3y1

[10] H. Devillez, N. Guyader, and A. Guerin-Dugue, "An eye fixation-related potentials analysis of the $\mathrm{P} 300$ potential for fixations onto a target object when exploring natural scenes," J Vis, vol. 15, no. 13, p. 20, 2015. [Online]. Available: https://www.ncbi.nlm.nih.gov/pubmed/26401627

[11] "BCI Competitions." [Online]. Available: http://www.bbci.de/competition/

[12] K. K. Ang, Z. Y. Chin, C. Wang, C. Guan, and H. Zhang, "Filter bank common spatial pattern algorithm on BCI competition IV datasets $2 \mathrm{a}$ and 2b," Frontiers in Neuroscience, vol. 6, pp. 1-9, 2012.

[13] P. Gaur, R. B. Pachori, H. Wang, and G. Prasad, "A multi-class EEGbased BCI classification using multivariate empirical mode decomposition based filtering and Riemannian geometry," Expert Systems with Applications, vol. 95, pp. 201-211, 2018.

[14] M. E. Sharbaf, A. Fallah, and S. Rashidi, "EEG-based multi-class motor imagery classification using variable sized filter bank and enhanced One Versus One classifier," 2nd Conference on Swarm Intelligence and Evolutionary Computation, CSIEC 2017 - Proceedings, pp. 135-140, 2017.

[15] R. T. Schirrmeister, J. T. Springenberg, L. D. J. Fiederer, M. Glasstetter, K. Eggensperger, M. Tangermann, F. Hutter, W. Burgard, and T. Ball, "Deep learning with convolutional neural networks for EEG decoding and visualization," Human Brain Mapping, vol. 38, no. 11, pp. 53915420, 2017.

[16] Y. Zhao, S. Yao, S. Hu, S. Chang, R. Ganti, M. Srivatsa, S. Li, and T. Abdelzaher, "On the Improvement of Classifying EEG Recordings Using Neural Networks," in IEEE International Conference on Big Data (BIGDATA) On, Boston, MA, USA, 2017, pp. 1709-1711.

[17] V. S. F.V.Stankevich, "Deep learning for BCI application," no. 1, pp. 48-55, 2017.

[18] K. Lafleur, K. Cassady, A. Doud, K. Shades, R. Eitan, and H. Bin, "Quadcopter control in three-dimensional space using a noninvasive motor imagery based brain-computer interface Karl," Journal of Neural Engineering, vol. 10, no. 4, pp. 1883-1889, 2013.

[19] D. J. Hand' and K. Yu2, "Idiot's Bayes-Not So Stupid After All?" Tech. Rep.

[20] E. Parzen, "On Estimation of a Probability Density Function and Mode," The Annals of Mathematical Statistics, 1962.

[21] C. Cortes, V. Vapnik, and L. Saitta, "Support-Vector Networks Editor," Tech. Rep., 1995.

[22] Kai Keng Ang, Zheng Yang Chin, Haihong Zhang, and Cuntai Guan, "Filter Bank Common Spatial Pattern (FBCSP) in Brain-Computer Interface," 2008 IEEE International Joint Conference on Neural Networks (IEEE World Congress on Computational Intelligence), pp. 2390-2397, 2008. [Online]. Available: http://ieeexplore.ieee.org/lpdocs/epic03/wrapper.htm?arnumber=4634130

[23] A. Rocha and S. K. Goldenstein, "Multiclass from binary: Expanding One-versus-all, one-versus-one and ECOC-based approaches," IEEE Transactions on Neural Networks and Learning Systems, vol. 25, no. 2, pp. 289-302, 2014.

[24] S. J. Yang, M. Berndl, D. M. Ando, M. Barch, A. Narayanaswamy, E. Christiansen, S. Hoyer, C. Roat, J. Hung, C. T. Rueden, A. Shankar, S. Finkbeiner, and P. Nelson, "Assessing microscope image focus quality with deep learning," BMC Bioinformatics, vol. 19, no. 1, 2018.

[25] H.-T. Zheng, J.-Y. Chen, X. Yao, A. Sangaiah, Y. Jiang, and C.-Z. Zhao, "Clickbait Convolutional Neural Network," Symmetry-Basel, vol. 10, no. 5, 2018. [Online]. Available: http://www.mdpi.com/2073$8994 / 10 / 5 / 138$

[26] S. Newatia and R. K. Aggerwal, "Convolutional Neural Network for ASR," in 2018 Second International conference on Electronics, Communication and Aerospace Technology (ICECA). IEEE, 2018, pp. 638642.

[27] S. Ioffe and C. Szegedy, "Batch Normalization: Accelerating Deep Network Training by Reducing Internal Covariate Shift,” 2015. [Online]. Available: http://arxiv.org/abs/1502.03167 
[28] N. Srivastava, G. Hinton, A. Krizhevsky, I. Sutskever, and R. Salakhutdinov, "Dropout: A Simple Way to Prevent Neural Networks from Overfitting," Journal of Machine Learning Research, vol. 15, pp. 19291958, 2014.

[29] D.-A. Clevert, T. Unterthiner, and S. Hochreiter, "Fast and Accurate Deep Network Learning by Exponential Linear Units (ELUs)," 2015.

[30] "Convolutional Neural Networks (CNNs / ConvNets)." [Online]. Available: https://cs231n.github.io/convolutional-networks/

[31] M. Khazi, A. Kumar, and M. J. Vidya, "Analysis of EEG Using 10 : 20 Electrode System,” vol. 1, no. 2, pp. 185-191, 2012.

[32] "BCI Competition IV: Results." [Online]. Available: http://www.bbci.de/competition/iv/results/

[33] G. Pfurtscheller and C. Neuper, "Motor imagery and direct braincomputer communication," Proceedings of the IEEE, vol. 89, no. 7, pp. 1123-1134, 2001.

[34] A. Palma, S. J. Møller, H. K. Iversen, and S. Puthusserypady, "An adaptive CSP filter to investigate user independence in a 3-class MIBCI paradigm," Comput. Biol. Med. (In Press), 2018. 